\title{
Determination of Volcanic Plume Three-Dimensional Structure Using CHRIS/PROBA-1 Multiangular Imagery: Case Study of Mount Etna
}

\author{
Sergio Baselga $\mathbb{D}^{1}{ }^{1}$ and Daniel Rodríguez-Pérez $\mathbb{D}^{2}$ \\ ${ }^{1}$ Departamento de Ingeniería Cartográfica, Geodesia y Fotogrametría, Universitat Politècnica de València, \\ Camino de Vera s/n, 46022 Valencia, Spain \\ ${ }^{2}$ Departamento de Física Matemática y de Fluidos, Facultad de Ciencias, Universidad Nacional de Educación a Distancia, \\ Paseo Senda del Rey 9, 28040 Madrid, Spain
}

Correspondence should be addressed to Sergio Baselga; serbamo@cgf.upv.es

Received 10 November 2017; Accepted 21 February 2018; Published 27 March 2018

Academic Editor: Paolo Addesso

Copyright (C) 2018 Sergio Baselga and Daniel Rodríguez-Pérez. This is an open access article distributed under the Creative Commons Attribution License, which permits unrestricted use, distribution, and reproduction in any medium, provided the original work is properly cited.

\begin{abstract}
The recent development of Earth observation satellites with multiangular capabilities and enhanced spectral resolution has led to preliminary attempts at determining the height of atmospheric scatterers, in particular, of top-cloud heights and smoke plumes originating from forest fires. Inspired by these previous studies, the present work presents an original methodology for the determination of the three-dimensional distribution of high-contrast atmospheric aerosols using multiangular images. The method starts with the approximately known geometry of image acquisition and a set of tie points and uses a linearized and regularized functional model to obtain the position of atmospheric scatterers identified by means of a semiassisted procedure on two or more images. A subsequent application to a CHRIS/PROBA-1 scene of Mount Etna following its eruption on June 14, 2014, allows determining the volcanic plume three-dimensional structure with a precision in the $100-200 \mathrm{~m}$ level.
\end{abstract}

\section{Introduction}

In the last two decades, Earth observation programmes have experienced a substantial development with the usage of sensors including not only increasing geometric resolution and number of radiometric bands but also multiangular capabilities. Among the missions and sensors providing multiangular images, one may find the Advanced Along-Track Scanning Radiometer (AATSR) onboard ENVISAT (active until 2012) and its predecessor ATSR-2, which provided images with $0^{\circ}$ and $+55^{\circ}$ along-track angles in seven radiometric bands (from $550 \mathrm{~nm}$ to $1200 \mathrm{~nm}$ ) to a spatial resolution of $1 \mathrm{~km}$. The Multiangle Imaging Spectroradiometer (MISR) onboard TERRA/SAR is capable of registering $0^{\circ}, 26.1^{\circ}, 45.6^{\circ}, 60.0^{\circ}$, and $70.5^{\circ}$ images in four spectral bands of the visible and near infrared with a spatial resolution of $275 \mathrm{~m}$ at nadir. Further, onboard the currently most recent mission, Sentinel3 (launched in February 2016), the sensor Sea and Land
Surface Temperature Radiometer (SLSTR) is designed to collect $0^{\circ}$ and $-55^{\circ}$ images with a spatial resolution of $300 \mathrm{~m}$ in the highest resolution mode. All of these sensors, however, are overcome in spatial resolution and number of radiometric bands by the Compact High Resolution Imaging Spectrometer (CHRIS) onboard ESA's PROBA-1 mission. CHRIS acquires images to a spatial resolution of $17 \mathrm{~m}$ (in acquisition mode 5 ) or $34 \mathrm{~m}$ (modes 1 to 4 ) in a large number of bands (62 in mode 1, 18 in modes 2, 3, and 4, and 37 in mode 5) for the spectral range of $400-1050 \mathrm{~nm}$ within nominal along-track angles of $0^{\circ}, \pm 36^{\circ}$, and $\pm 55^{\circ}$ (see [1]).

The most significant benefit obtained from the use of multiangular images versus the traditional use of only nadiral images derives from a better characterization of the spectral response of the surface appearing in the scene without the use of additional prior hypothesis [2-4]. Some few works, however, use this multiangular capability to retrieve information on the vertical distribution of scatterers in the atmosphere, 
albeit with little detailed results (i.e., poor vertical resolution) and just for significantly dense scatterers. Hence, Horváth and Davies [5] and Moroney et al. [6] determined top-cloud heights, and Kahn et al. [7] obtained heights of smoke plumes due to forest fires. Theoretical radiative transfer and diffusion models have also been the topic of recent research (e.g., $[8,9])$. Since knowledge of the distribution of aerosols in the atmosphere has been pointed out by the Intergovernmental Panel on Climate Change (IPCC) as one of the key aspects for a better understanding of the climate evolution mechanisms [10], it seems that research on the application of remote sensing imagery to this question must be fostered. There is no doubt that satellite imagery has been increasingly used in recent years for aerosol retrieval; however, its main application has remained in the context of determination of aerosol general parameters (e.g., Aerosol Optical Depths) having left the determination of vertical distribution, with very little exceptions, to other techniques like LIDAR from the CALIPSO (Cloud-Aerosol Lidar and Infrared Pathfinder Satellite Observation) programme $[11,12]$.

In this paper, we present a methodology for the determination of the three-dimensional structure of high-contrast aerosols in the atmosphere using multiangular images of the CHRIS/PROBA-1 sensor along with its application to the determination of the vertical distribution of Mount Etna's volcanic plume. In the following section, we explain the data preparation and the functional and stochastic models that are input to the adjustment by the least-squares method. Then we will present and discuss the results and draw the corresponding conclusions, including the limitations of the proposed methodology and possible lines for future research.

\section{Materials and Methods}

2.1. Data Preparation. We use the VISAT-BEAM opensource toolbox [13] that ESA released for public analysis, processing, and exploitation of their products to work with the CHRIS images of Mount Etna dated June 28, 2014-subsequent to its eruption on June 14, 2014-which are available at the ESA download site (ESA Earth Observation Users' Single Sign On, https://eo-sso-idp.eo.esa.int/idp/ umsso20/admin), where the volcanic plume is still clearly visible. The image files show the scenes corresponding to along-track nominal angles of $0^{\circ},+36^{\circ},+55^{\circ}$, and $-55^{\circ}$ (the image file corresponding to $-36^{\circ}$ is missing). Further, it has to be noted that the $-55^{\circ}$ scene contains little information useful for the determination of the three-dimensional structure of the plume: a small portion of the plume with too little features whose homologous points could be identified in the other scenes; therefore, we decided not to include it in the analysis. A note of caution is worth here regarding the angular value: $+55^{\circ},+36^{\circ}$, and $0^{\circ}$ are only the nominal along-track angle values of CHRIS/PROBA, while the real ones may be actually different due to the across-track pointing of the platform. While we will be continuously referring to $+55^{\circ},+36^{\circ}$, and $0^{\circ}$ scenes, these values cannot be entered in the subsequent computations as true values of the image geometry, which indeed involves along-track as well as across-track angular

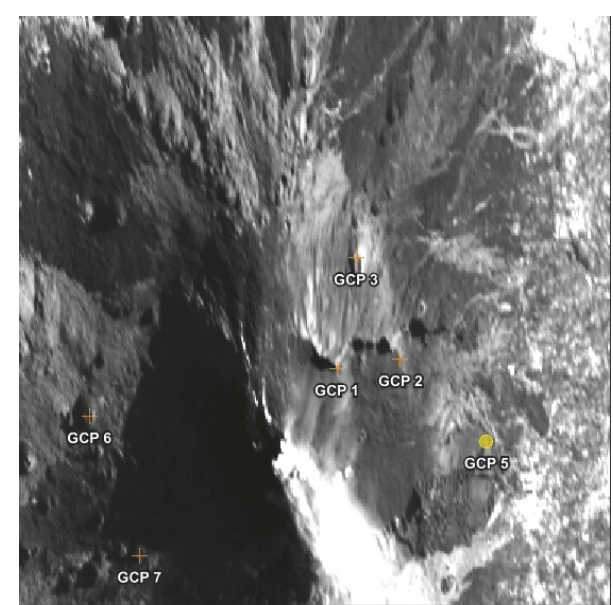

Figure 1: GCPs over the $55^{\circ}$ scene (VISAT-BEAM).

values. At any rate, knowledge of the actual values for these angles will not be needed for our calculation.

For every image, its metadata include an estimation of the image center time (here 5:06:42, 5:05:55, and 5:05:07 for $0^{\circ},+36^{\circ}$, and $+55^{\circ}$ scenes, resp.). We use these times to interpolate in the corresponding telemetry file that can be retrieved from ESA's REDU Center (http://194.78.233.110/ products/data/CHRIS_Additional_data/) and compute the WGS84 Cartesian coordinates for every image center.

CHRIS images usually suffer from a misalignment problem of unknown origin, which makes the image centers differ considerably from their targeted positions $[4,14]$. Besides the inclusion into VISAT-BEAM of the corresponding telemetry file, some ground control points (GCPs) also have to be supplied in order to correct image misalignments. Apart from these, some additional ground points with known coordinates were used to evaluate the quality of the resulting transformation. We used Google Earth to retrieve the terrain coordinates of all ground points. We found the resulting typical errors to be around $100 \mathrm{~m}$ for the $0^{\circ}$ and $36^{\circ}$ images, whereas 200 to $300 \mathrm{~m}$ typical errors were found for the $55^{\circ}$ image. This is likely to be caused by greater point identification difficulties in a very inclined image. As an example, the distribution of the selected GCPs over the $55^{\circ}$ scene, and their corresponding coordinates, is given in Figure 1 and Table 1.

Next we identified homologous points in the different images of the plume using one radiometric band, where the contrast is high (band 26, 682.2 nm). It is worth noting that other studies of volcanic plumes have used spectral bands of very similar wavelengths (e.g., [15]). Zooming in and out over the images by means of the VISAT-BEAM, we can be quite accurate in identifying some homologous points in the different images and obtaining their coordinates, especially for the plume outline. For other parts in the plume, however, the identification of homologous points turned out to be almost impossible. Figures 2-4 show general layouts of the set of homologous points (accurate identification was done in zoomed-in views).

The larger limitation in identifying homologous points over the $55^{\circ}$ image is self-evident: some of the homologous 
TABLE 1: Image and terrain coordinates of GCPs for the $55^{\circ}$ scene.

\begin{tabular}{lcccc}
\hline Point number & $x$-image & $y$-image & Longitude $\left(^{\circ}\right)$ & Latitude $\left(^{\circ}\right)$ \\
\hline$(1)$ & 200.5 & 224.5 & 15.024593 & 37.756615 \\
$(2)$ & 240.5 & 218.5 & 15.038412 & 37.75333 \\
$(3)$ & 212.5 & 154.5 & 15.039819 & 37.771614 \\
$(5)$ & 294.5 & 270.5 & 15.089861 & 37.713634 \\
$(6)$ & 45.0 & 254.5 & 14.919533 & 37.75655 \\
$(7)$ & 76.5 & 342.5 & 14.934227 & 37.725693 \\
\hline
\end{tabular}

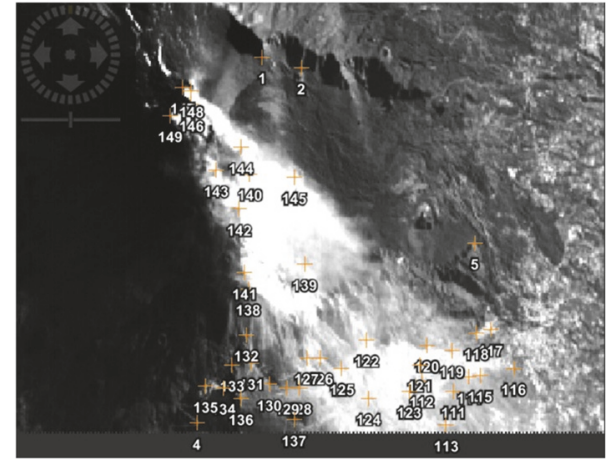

FIgURE 2: Homologous points for the $0^{\circ}$ scene (VISAT-BEAM).

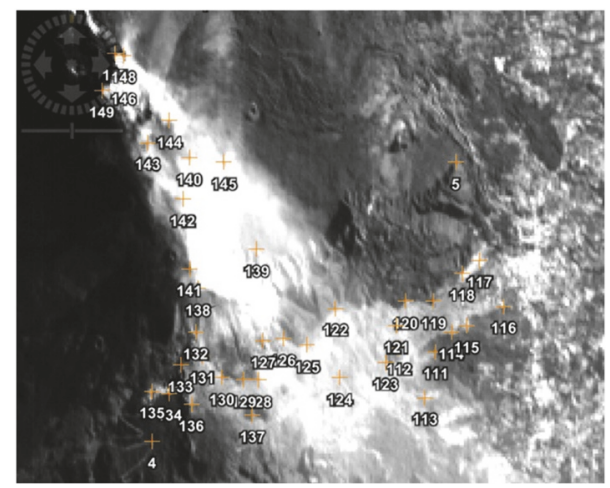

FIGURE 3: Homologous points for the $36^{\circ}$ scene (VISAT-BEAM).

points in the two other images are out of scene now; other points were included albeit with a limited degree of certainty about their correctness, while some others were more reliable.

It has to be noted that these coordinates correspond to the plume projection onto the terrain for every image. As appearing in Figure 5 in the next subsection, these coordinates will be denoted with lowercase letters $x, y$, and $z$.

2.2. Functional and Stochastic Models. For the sake of simplicity, we derive now the functional model based on two images. Its generalization to three or more images, to be used later, is straightforward.

After interpolation in the telemetry file, the satellite positions for the two scene shots are assumed to be known (at least approximately) in a global geodetic system like the WGS84. A right-hand set of Cartesian coordinates is defined in this system with $z$-axis in direction of the (conventional) Earth rotation axis and $x$-axis and $y$-axis in the equatorial plane (with $x$-axis pointing along the direction of $0^{\circ}$ longitude). Transformation from geodetic coordinates latitude $\varphi$, longitude $\lambda$, and height over the reference ellipsoid $h$ to Cartesian coordinates $x, y$, and $z$ is obtained applying the well-known formulae [16-18]

$$
\begin{aligned}
& x=(\nu+h) \cos \varphi \cos \lambda, \\
& y=(\nu+h) \cos \varphi \sin \lambda, \\
& z=\left(\nu\left(1-e^{2}\right)+h\right) \sin \varphi,
\end{aligned}
$$

where $v$ is the curvature radius of the ellipsoid in the prime vertical:

$$
v=\frac{a}{\sqrt{1-e^{2} \sin ^{2} \varphi}}
$$

and $a$ is the semimajor axis of the ellipsoid, whose eccentricity is denoted by $e$. The relationship between ellipsoidal height $h$ and orthometric height $H$ (i.e., above the geoid or mean sea level) is also straightforward,

$$
h=H+N \text {, }
$$

through knowledge of the geoid undulation $N$, here assumed to be constant for the entire study area $N=43.8 \mathrm{~m}$ as taken from the EGM2008 geoid [19].

For a particular scatterer in the atmosphere of unknown coordinates $(X, Y, Z)$, we have the projected point onto the terrain of coordinates $\left(x_{1}, y_{1}, z_{1}\right)$ for image 1 and $\left(x_{2}, y_{2}, z_{2}\right)$ for image 2 . These coordinates are known after direct measurement of image coordinates and terrain georeferencing under VISAT-BEAM and later transformation from geodetic coordinates to Cartesian coordinates with the formulas above. We also have the corresponding satellite coordinates $\left(X_{1}, Y_{1}, Z_{1}\right)$ and $\left(X_{2}, Y_{2}, Z_{2}\right)$ obtained after interpolation using the telemetry file, and the coordinates of the scene target $\left(X_{T}, Y_{T}, Z_{T}\right)$. The corresponding geometry is shown in Figure 5. tions,

Projective rays fulfill the straight-line parametric equa-

$$
\begin{aligned}
& X=X_{i}+\mu_{i}\left(x_{i}-X_{i}\right), \\
& Y=Y_{i}+\mu_{i}\left(y_{i}-Y_{i}\right), \\
& Z=Z_{i}+\mu_{i}\left(z_{i}-Z_{i}\right),
\end{aligned}
$$




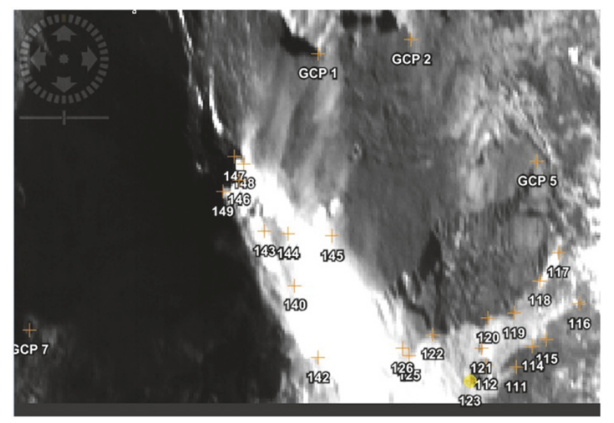

FIgURE 4: Homologous points for the $55^{\circ}$ scene (VISAT-BEAM).

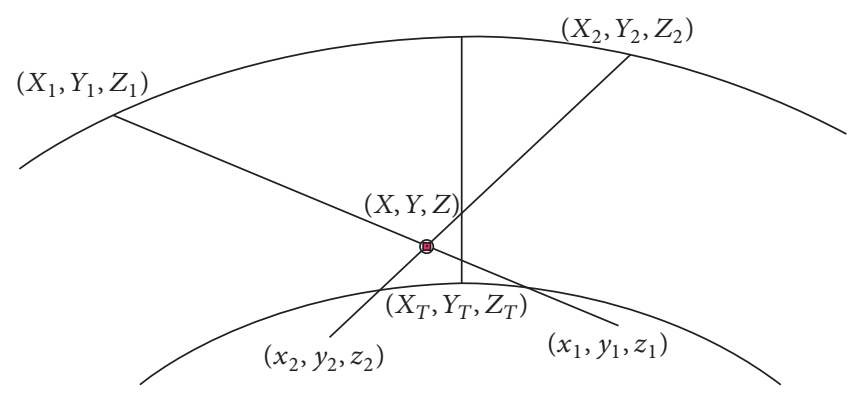

FIGURE 5: Geometry of projective rays for a scatterer in the atmosphere.

for every image $i$ with the respective parameter $\mu_{i}$. The coordinates of the scatterer $(X, Y, Z)$ are common to both sets of equations and therefore can be determined with two or more images.

For simplicity, we can work with the first of these equations only and denote it after a slight rearrangement as

$$
F\left(X_{i}, \mu_{i}, x_{i}, X\right)=\frac{X}{\mu_{i}}-\frac{X_{i}}{\mu_{i}}-x_{i}+X_{i}=0,
$$

with $F$ being the functional model of the problem at hand. This is an equation that is exactly fulfilled with the exact values of satellite coordinate in scene $i, X_{i}$, straight-line parameter $\mu_{i}$, terrain coordinate $x_{i}$, and scatterer coordinate $X$. We could be naive enough to think that we truly know three of these parameters (the satellite position is known by means of the telemetry, the image coordinate is measured, and parameter of the straight line is defined through the coordinates of these two points belonging to the line) so that there is only one unknown, coordinate $X$. However, these three parameters are not known with total accuracy so that, if simply fixed, the inaccuracies in their values would be transferred to inaccuracies in the final coordinate $X$ with no possible way to separate the errors corresponding to each of the error sources in addition to causing an incontrollable bias in the solution. We must acknowledge, however, that they have been obtained only approximately: the satellite coordinate $X_{i}$ was computed after interpolation for the image central time; terrain coordinate $x_{i}$ is the result of a measurement and it is affected by the inevitable random error that is present in every measurement process (including also the previous measurements needed for the image georeferencing); and, finally, parameter $\mu_{i}$ can be roughly determined taking into account the approximate scene inclination $\left(0^{\circ}, 36^{\circ}\right.$, or $\left.55^{\circ}\right)$ or more accurately from the use of these (approximate) satellite and terrain coordinates. To remedy this, we will use system regularization that accounts for the estimated error size in the (inaccurately) known parameters and has the effect of fixing the known parameters albeit with a loose margin, that is, not as exact values but as statistical values each with a given most probable value and a given standard deviation so that the inaccuracies in the approximate parameters are not transferred to the final solution.

Consider, first, the fact that the functional model (see (5)) is not linear in all variables; second, consider the fact that approximate values for all these variables may be obtained (they will be denoted as $X_{i 0}, \mu_{i 0}, X_{0}$, and $x_{i 0}$ ), and, third, consider that the disparate absolute degree of accuracy of these approximate values will require the system to be regularized; linearization of the model is therefore necessary. After firstdegree Taylor's expansion, the functional model reads

$$
\begin{aligned}
F( & \left.X_{i}, \mu_{i}, x_{i}, X\right) \\
= & F\left(X_{i 0}+\delta X_{i}, \mu_{i 0}+\delta \mu_{i}, x_{i 0}+r_{x i}, X_{0}+\delta X\right) \\
\approx & F\left(X_{i 0}, \mu_{i 0}, x_{i 0}, X_{0}\right)+\left[\frac{\partial F}{\partial X_{i}}\right]_{0} \delta X_{i}+\left[\frac{\partial F}{\partial \mu_{i}}\right]_{0} \delta \mu_{i} \\
& +\left[\frac{\partial F}{\partial x_{i}}\right]_{0} r_{x i}+\left[\frac{\partial F}{\partial X}\right]_{0} \delta X \approx 0,
\end{aligned}
$$


where partial derivatives are to be evaluated for the approximate values, $\delta X_{i}, \delta \mu_{i}$, and $\delta X$ denote corrections to the corresponding approximate values $X_{i 0}, \mu_{i 0}$, and $X_{0}$, and $r_{x i}$ denotes the residual to be determined after the adjustment for the observation $x_{i 0}$. After linearization, we have transformed the problem into the determination of the corrections $\delta X_{i}, \delta \mu_{i}$, and $\delta X$ to approximate values $X_{i 0}, \mu_{i 0}$, and $X_{0}$ as well as the residual $r_{x i}$ of the observed value $x_{i 0}$. We will then obtain the final adjusted values as

$$
\begin{aligned}
& X=X_{0}+\delta X, \\
& X_{i}=X_{i 0}+\delta X_{i}, \\
& \mu_{i}=\mu_{i 0}+\delta \mu_{i}, \\
& x_{i}=x_{i 0}+r_{x i} .
\end{aligned}
$$

A

$$
\begin{aligned}
& \begin{array}{rr}
1-\frac{1}{\mu_{10}^{(1)}} & \\
& 1-\frac{1}{\mu_{10}^{(1)}}
\end{array} \\
& 1-\frac{1}{\mu_{10}^{(1)}} \\
& 1-\frac{1}{\mu_{20}^{(1)}} \\
& 1-\frac{1}{\mu_{20}^{(1)}} \\
& = \\
& 1-\frac{1}{\mu_{10}^{(2)}} \\
& 1-\frac{1}{\mu_{10}^{(2)}} \\
& 1-\frac{1}{\mu_{10}^{(2)}} \\
& 1-\frac{1}{\mu_{20}^{(2)}} \\
& 1-\frac{1}{\mu_{20}^{(2)}} \\
& 1-\frac{1}{\mu_{20}^{(2)}}
\end{aligned}
$$

Computing the derivatives and rearranging the equation to leave the unknowns in the left-hand side, except for the residual term, we can write

$$
\begin{gathered}
\left(1-\frac{1}{\mu_{i 0}}\right) \delta X_{i}+\frac{X_{i 0}-X_{0}}{\mu_{i 0}^{2}} \delta \mu_{i}+\frac{1}{\mu_{i 0}} \delta X \\
=x_{i 0}+\frac{X_{i 0}}{\mu_{i 0}}-\frac{X_{0}}{\mu_{i 0}}-X_{i 0}+r_{x i} .
\end{gathered}
$$

Analogous expressions can be obtained for the $Y$ and $Z$ coordinates. For two images (denoted with subscripts 1 and 2 ), considering the coordinates of several scatterers (denoted with superscripts (1), (2), etc.), we can finally write the corresponding system of equations in matrix form as

$$
\mathbf{A x}=\mathbf{k}+\mathbf{r}
$$

with

\section{,}


so on. Similarly, rows are generated in blocks of 6 for every scatterer.

Vectors $\mathbf{x}, \mathbf{k}$, and $\mathbf{r}$ have the following structure:

$$
\mathbf{x}=\left(\begin{array}{l}
\delta X_{1} \\
\delta Y_{1} \\
\delta Z_{1} \\
\delta X_{2} \\
\delta Y_{2} \\
\delta Z_{2} \\
\delta \mu_{1}^{(1)} \\
\delta \mu_{2}^{(1)} \\
\delta X^{(1)} \\
\delta Y^{(1)} \\
\delta Z^{(1)}
\end{array}\right.
$$

Rectangular matrix $\mathbf{A}$ and column vector $\mathbf{k}$ are made of numeric values that can be computed from the approximate or measured values, whereas column vectors $\mathbf{r}$ (observation residuals) and $\mathbf{x}$ (correction to approximate values) are the unknowns to be determined in the adjustment. Before regularization, to be explained later on, we have, for $n$ scatterers, $6 n$ equations with $6+5 n$ unknowns for the case of a model based on 2 images, $9 n$ equations with $9+6 n$ unknowns for a model based on 3 images, and so forth.

The resulting Cartesian coordinates can be transformed back to geodetic coordinates by making use of the wellknown formulas [17],

$$
\begin{aligned}
& \varphi=\arctan \frac{Z+e^{\prime 2} b \sin ^{3} \theta}{p-e^{2} a \cos ^{3} \theta}, \\
& \lambda=\arctan \frac{Y}{X}, \\
& h=\frac{p}{\cos \varphi}-\nu,
\end{aligned}
$$

in terms of the following auxiliary variables and the curvature radius already defined in (2):

$$
\begin{aligned}
\theta & =\arctan \frac{Z a}{p b}, \\
e^{\prime 2} & =\frac{a^{2}-b^{2}}{b^{2}}, \\
p & =\sqrt{X^{2}+Y^{2}},
\end{aligned}
$$

where $b$ is the semiminor axis of the ellipsoid. We can therefore obtain the ellipsoidal height $h$ or, by means of (3), the height above mean sea level $H$.

Now the system of equations in (9) has to be adjusted by least-squares method, since we assume the measurands 
to be statistical variables following normal distributions. A reasonable a priori estimate for the precision of each terrain coordinate can be $100 \mathrm{~m}$ for the $0^{\circ}$ and $36^{\circ}$ images and $200 \mathrm{~m}$ for the $55^{\circ}$ image, in accordance with the georeferencing analysis previously mentioned. This also goes along the lines of Alonso and Moreno [14] who found georeferencing errors to be around $100 \mathrm{~m}$ for flat areas and up to $300 \mathrm{~m}$ for abrupt reliefs. Once defined in this fashion, the standard error $\sigma$ of each measured terrain coordinate, a diagonal weight matrix $\mathbf{W}$ (with dimension equal to the number of rows in $\mathbf{A}$ ) is created with weights in its diagonal, with each of them defined as

$$
w=\frac{1}{\sigma^{2}} .
$$

Further, the system of equations in (9) has unknowns of very different nature for which we expect very different values: corrections to the approximate straight-line parameters, $\delta \mu_{1}{ }^{(1)}$, and so forth; satellite coordinates, $\delta X_{1}$, and so forth; and position of the scatterers, $\delta X^{(1)}$, and so forth. The application of parameter regularization to the system of equations is interesting then, as some other authors [3] have already pointed out for the case of multiangular images.

We pay attention now to the computation of the different approximate values and their corresponding degrees of accuracy, which will define the figures to use in the regularization. First, we have assumed that all pixels of each image are simultaneously acquired; obviously, this is only a working assumption, especially since CHRIS/PROBA uses a nonstandard push-broom acquisition technique with a nonuniform rotation speed of the platform in order to maximize image quality. However, we can explain the validity of this simplified hypothesis as a working assumption for the final $3 \mathrm{D}$ reconstruction of the plume as follows. Taking into account the fact that the time difference with respect to the central time in each scene can amount to $10 \mathrm{~s}$ [14] and the satellite is orbiting the Earth $600 \mathrm{~km}$ above its surface [1] with an orbital period of $100 \mathrm{~min}$ [1], the differences in the satellite positions during the image acquisition can amount to $70 \mathrm{~km}$. Previous studies indicate that there are already differences of the order of several $\mathrm{km}$ between satellite coordinates obtained by different techniques [14]. All in all, as they say, these discrepancies usually have a negligible effect on the final solution. As an example, we can reckon that an error of $70 \mathrm{~km}$ in the satellite position may represent in the worst case only some $115 \mathrm{~m}$ of error in the position of a scatterer located $1 \mathrm{~km}$ above the Earth's surface. We therefore estimate as $50 \mathrm{~km}$ the typical error of the initial approximate coordinates of the satellite (assumed to be static during acquisition) and understand that the impact on the final results (typically of the order of tens of meters) is acceptable for the definition of the plume 3D structure.

Another working assumption is the static character assumed for the plume during the successive image acquisitions. Obviously, the effect of the wind modifies the shape of the plume, although it can be argued that the relative displacement between points belonging to the plume will be more than one degree of magnitude less than the total wind speed. For instance, a wind speed of $10 \mathrm{~km} / \mathrm{h}(2.8 \mathrm{~m} / \mathrm{s})$ represents some $130 \mathrm{~m}$ of wind displacement between two consecutive images acquired with some $47 \mathrm{~s}$ time separation, surely leading to a relative displacement of points in the plume below the level of some 10-20 $\mathrm{m}$. There are also other intrinsic movements in the plume, due to thermal turbulent convection, which would be difficult to accurately model; however, we can also assume them to be below the level of a few tens of meters for our time intervals. A simulation using a mesoscale numerical model [20] agrees with the order of magnitude of this velocity.

Regarding the coordinates of a particular scatterer in the atmosphere, we can use as a first approximation their planimetric coordinates $(\varphi, \lambda)$ in the nominal along-track $0^{\circ}$ image along with a reasonable average height (e.g., the target height). We estimate the typical corrections to this value as some $500 \mathrm{~m}$.

Finally, straight-line parameters can be computed from the scene shot geometry or, more precisely, in terms of the satellite and scatterer approximate coordinates. Their values result from the order of unity and the corresponding corrections to be obtained may be of the order of 0.05 .

We will therefore perform the regularization of the system of equations by adding one pseudoobservation equation in matrix $\mathbf{A}$ for every unknown. These regularization equations will be of the type (in this example for coordinate $X_{1}$ )

$$
\delta X_{1}=0+r_{\delta X_{10}}
$$

and will be accompanied by the corresponding weight in the diagonal matrix $\mathbf{W}$ :

$$
w X_{10}=\frac{1}{\sigma_{X_{10}}{ }^{2}},
$$

where $\sigma_{X_{10}}=50000 \mathrm{~m}$ is the estimated error for satellite coordinates, as mentioned previously.

The resulting system of equations is solved by leastsquares method as

$$
\begin{aligned}
& \mathbf{x}=\left(\mathbf{A}^{\mathrm{T}} \mathbf{W A}\right)^{-1} \mathbf{A}^{\mathrm{T}} \mathbf{W} \mathbf{k}, \\
& \mathbf{r}=\mathbf{A} \mathbf{x}-\mathbf{k} .
\end{aligned}
$$

The a posteriori unit weight standard deviation is obtained [21] as

$$
\widehat{\sigma}_{0}=\sqrt{\frac{\mathbf{r}^{T} \mathbf{W r}}{m-n}},
$$

where $m$ and $n$ are, respectively, the number of equations and the number of unknowns. The proximity of the value to unity is an indicator of the quality of the adjustment (correctness of functional and stochastic models). We can also apply the data snooping test [21] to determine the homologous points that were incorrectly identified.

\section{Results and Discussion}

After adjustment of the model based on two images $\left(0^{\circ}\right.$ and $36^{\circ}$ ), we obtain residuals typically below $100 \mathrm{~m}$ with a few 


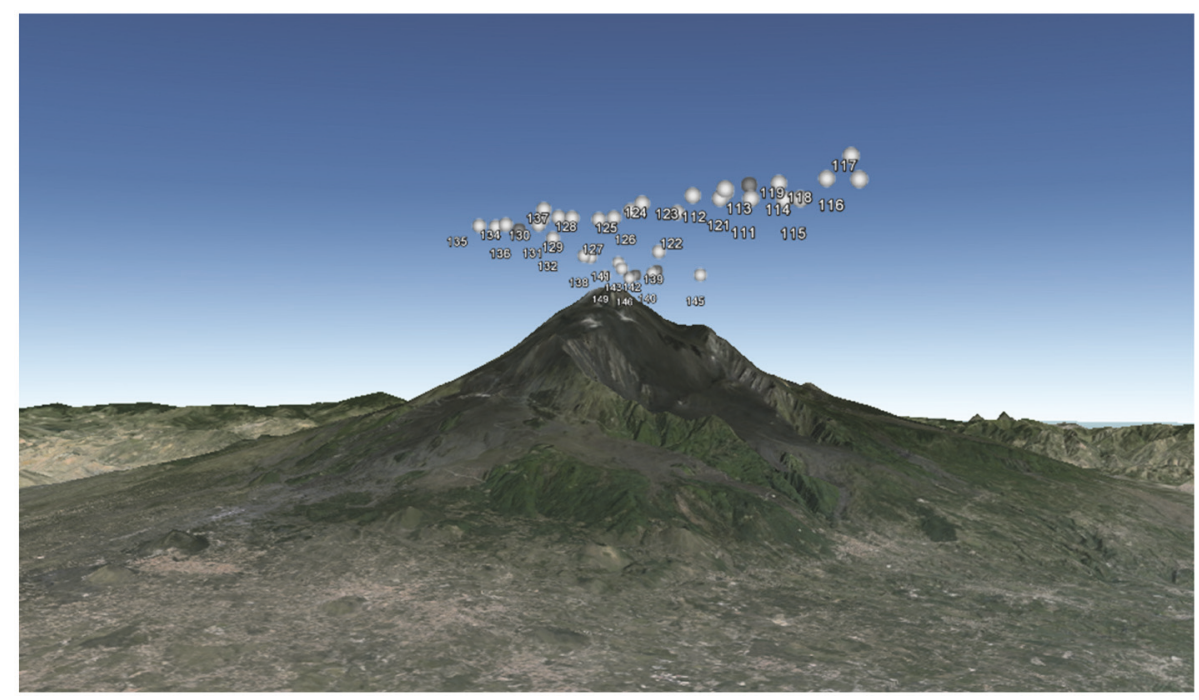

Figure 6: Representation of the distribution of scatterers over a Google Earth view (@ 2015 Google Inc., used with permission. Google and the Google logo are registered trademarks of Google Inc.).

exceptions for points $140,142,144,145$, and 146 reaching 150-200 m, which can be regarded as acceptable results (they are consistent with the prior precision). The a posteriori unit weight standard deviation is 0.914 , and its closeness to unity confirms the validity of the adjustment. Iteration of the computations produces significant changes in neither the residuals nor the coordinates of the scatterers.

Regarding the adjustment of the model based on three images $\left(0^{\circ}, 36^{\circ}\right.$, and $\left.55^{\circ}\right)$, we start by noticing an undesired result: the a posteriori unit weight standard deviation is now 2.998, which indicates that the existing errors are 3 times, on average, the expected value. By inspecting the corresponding residuals, we find out that some of them have been affected by gross errors. They correspond to some of the doubtful observations we made over the third image $\left(55^{\circ}\right)$. Starting from the largest residual (corresponding to point 142 on image 3), we iteratively eliminate the point with the worst residual in the successive adjustments as it is customary in the data snooping procedure until all the remaining residuals are acceptable. The a posteriori unit weight standard deviation is now 1.870 and the adjustment can be accepted. We observe, however, that the final residuals are mostly below $100 \mathrm{~m}$ for the $0^{\circ}$ and $36^{\circ}$ images, whereas they have a typical size of $400-500 \mathrm{~m}$ for the $55^{\circ}$ images. This leads us to conclude that the error estimation for $0^{\circ}$ and $36^{\circ}$ images $(\sigma=100 \mathrm{~m})$ was correct, whereas the error estimation for the $55^{\circ}$ image $(\sigma=200 \mathrm{~m})$ was too optimistic. At any rate, the resulting coordinates of the scatterers do not differ much from those obtained with the two-image model so that both adjustments can be regarded as valid. These differences are mostly below $60 \mathrm{~m}$, being smaller in the vertical components than in the horizontal ones, amounting to some $200 \mathrm{~m}$ for the worst case. It all can be regarded as a sensible result if we take into account the different uncertainties involved in the model.

The three-dimensional distribution of the particular scatterers defined in the plume contour is shown over a Google
Earth view in Figure 6. Although these scatterers constitute only a small sample of the volcanic plume, they offer the possibility to characterize its three-dimensional distribution including height, shape, and direction (towards southeast).

As a final comment, we can recall that the best observational geometry for the determination of coordinates by intersection is the one that is made at a right angle in the unknown object [22]. This means that, to optimally define the vertical structure of the plume by using only two images, we would need images at $45^{\circ}$ and $-45^{\circ}$. If more than two images are to be acquired, the corresponding angles should be more or less evenly separated from this value on both sides. The design of the CHRIS/PROBA-1 nominal angles (with $45^{\circ}$ almost halfway from $36^{\circ}$ and $55^{\circ}$ ) closely conforms to this idea and must be regarded as nearly optimal, at least from the purely geometrical point of view, obviating the fact that identification of homologous points is more difficult the larger the view angle difference is.

\section{Conclusions}

An original methodology for the determination of the three-dimensional distribution of high-contrast atmospheric scatterers using multiangular images was derived and subsequently applied to the case of Mount Etna's volcanic plume from high-resolution CHRIS/PROBA-1 images. The final results proved to be reliable within the 100-200 m level.

The methodology presents, however, some limitations in its different steps. Regarding data acquisition, a high degree of contrast of aerosols in the atmosphere is needed to perform the necessary identification of homologous points in the different inclination scenes. The correct identification of these homologous points is a critical factor limiting the precision of the final results. Gross error testing and identification are therefore unavoidable. Another limiting factor comes from the relatively small sample of identifiable features appearing 
on all the different views of the plume. These limited numbers of homologous points may suffice, however, to roughly define the three-dimensional distribution of the plume. With regard to the different simplifications in the computations, we note the assumption that the plume does not move or change its shape during the total acquisition time, as well as the simplification that for every image all pixels have been acquired in a common time. Both may lead to errors assumed to be within the indicated total error budget (100-200 m). Finally, from the algebraic point of view, we may note that the problem is ill-conditioned and requires the use of regularization. It is expected that application to other cases where more images are available (the five of them or at least images on both sides of the nadir view) produces better results than those obtained here only with $0^{\circ},+36^{\circ}$, and $+55^{\circ}$ images and would allow drawing conclusions on the measuring precision achievable using different sets of angles.

Further research may include application to other areas and types of dense aerosols (e.g., wildfire plumes), automated measuring of homologous points over the different images, inclusion of a more detailed characterization of the platform movement during the acquisition, and the use of robust estimation to detect incorrect measurements.

\section{Conflicts of Interest}

The authors declare that there are no conflicts of interest regarding the publication of this paper.

\section{References}

[1] Surrey Satellite Technology Ltd, "CHRIS data format. Doc. No. 0114848," 2008, https://earth.esa.int/c/document_library/ get_file?folderId=23844\&amp;name=DLFE-592.pdf.

[2] L. S. Galvão, F. J. Ponzoni, V. Liesenberg, and J. R. D. Santos, "Possibilities of discriminating tropical secondary succession in Amazônia using hyperspectral and multiangular CHRIS/PROBA data," International Journal of Applied Earth Observation and Geoinformation, vol. 11, no. 1, pp. 8-14, 2009.

[3] W. M. F. Grey and P. R. J. North, "Aerosol optical depth from dual-view (A)ATSR satellite observations," in Satellite Aerosol Remote Sensing over Land, A. A. Kokhanovsky and G. de Leeuw, Eds., Springer, Berlin, Germany, 2009.

[4] W. H. Davies and P. R. J. North, "Synergistic angular and spectral estimation of aerosol properties using CHRIS/PROBA1 and simulated Sentinel-3 data," Atmospheric Measurement Techniques, vol. 8, no. 4, pp. 1719-1731, 2015.

[5] Á. Horváth and R. Davies, "Feasibility and error analysis of cloud motion wind extraction from near-simultaneous multiangle MISR measurements," Journal of Atmospheric and Oceanic Technology, vol. 18, no. 4, pp. 591-608, 2001.

[6] C. Moroney, R. Davies, and J.-P. Muller, "Operational retrieval of cloud-top heights using MISR data," IEEE Transactions on Geoscience and Remote Sensing, vol. 40, no. 7, pp. 1532-1540, 2002.

[7] R. A. Kahn, W.-H. Li, C. Moroney, D. J. Diner, J. V. Martonchik, and E. Fishbein, "Aerosol source plume physical characteristics from space-based multiangle imaging," Journal of Geophysical Research: Atmospheres, vol. 112, no. 11, Article ID D11205, 2007.
[8] Y. Yuan, X. Huang, Y. Shuai, and Q.-J. Mao, "Study on the Influence of Aerosol Radiation Balance in One-Dimensional Atmospheric Medium Using Pn-Approximation Method," Mathematical Problems in Engineering, vol. 2014, Article ID 767231, 2014.

[9] C.-G. Zhu, C.-X. Lü, and J. Wang, "Evaluation of aerosol fire extinguishing agent using a simple diffusion model," Mathematical Problems in Engineering, vol. 2012, Article ID 873840, 2012.

[10] Intergovernmental Panel on Climate Change, Climate Change 2013-The Physical Science Basis. Contribution of Working Group I to the Fifth Assessment Report of the Intergovernmental Panel on Climate Change, T. F. Stocker, D. Qin, G.-K. Plattner, Eds., Cambridge University Press, Cambridge, UK, 2013.

[11] R. Lasaponara, "Geospatial analysis from space: Advanced approaches for data processing, information extraction and interpretation," International Journal of Applied Earth Observation and Geoinformation, vol. 20, no. 1, pp. 1-3, 2012.

[12] Y. Wu, L. Cordero, B. Gross, F. Moshary, and S. Ahmed, "Assessment of CALIPSO attenuated backscatter and aerosol retrievals with a combined ground-based multi-wavelength lidar and sunphotometer measurement," Atmospheric Environment, vol. 84, pp. 44-53, 2014.

[13] Brockmann Consult, "BEAM toolbox," 2011, http://www.brockmann-consult.de/beam.

[14] L. Alonso and J. Moreno, "Advances and limitations in a parametric geometric correction of CHRIS/PROBA data," in Proceedings of the in Proceedings of the 3rd ESA CHRIS/Proba workshop, Frascati, Italy, 2005.

[15] I. M. Watson, V. J. Realmuto, W. I. Rose et al., “Thermal infrared remote sensing of volcanic emissions using the moderate resolution imaging spectroradiometer," Journal of Volcanology and Geothermal Research, vol. 135, no. 1-2, pp. 75-89, 2004.

[16] P. Vanicek and E. J. Krakiwsky, Geodesy: the concepts, Elsevier, Amsterdam, The Netherlands, 2nd edition, 1986.

[17] G. P. Gerdan and R. E. Deakin, "Transforming cartesian coordinates $\mathrm{X}, \mathrm{Y}, \mathrm{Z}$ to geographical coordinates $\varphi, \lambda$, h," Australian Surveyor, vol. 44, no. 1, pp. 55-63, 1999.

[18] C. Jekeli, eometric Reference Systems in Geodesy, Division of Geodetic Science, School of Earth Sciences, Ohio State University, USA, 2008.

[19] N. K. Pavlis, S. A. Holmes, S. C. Kenyon, and J. K. Factor, "The development and evaluation of the Earth Gravitational Model 2008 (EGM2008)," Journal of Geophysical Research: Solid Earth, vol. 117, no. 4, pp. 1978-2012, 2012.

[20] M. Favalli, F. Mazzarini, M. T. Pareschi, and E. Boschi, "Role of local wind circulation in plume monitoring at Mt. Etna volcano (Sicily) : Insights from a mesoscale numerical model," Geophysical Research Letters, vol. 31, no. 9, pp. L09105-4, 2004.

[21] C. D. Ghilani, "Adjustment Computations," in Spatial Data Analysis, John Wiley \& Sons, Inc, Hoboken, NJ, USA, 5th edition, 2010.

[22] J. McCormac, Surveying Fundamentals, Prentice-Hall, Inc., Englewood Cliffs, NJ, USA, 1983. 


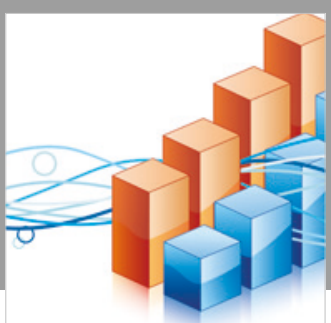

Advances in

Operations Research

\section{-n-m}
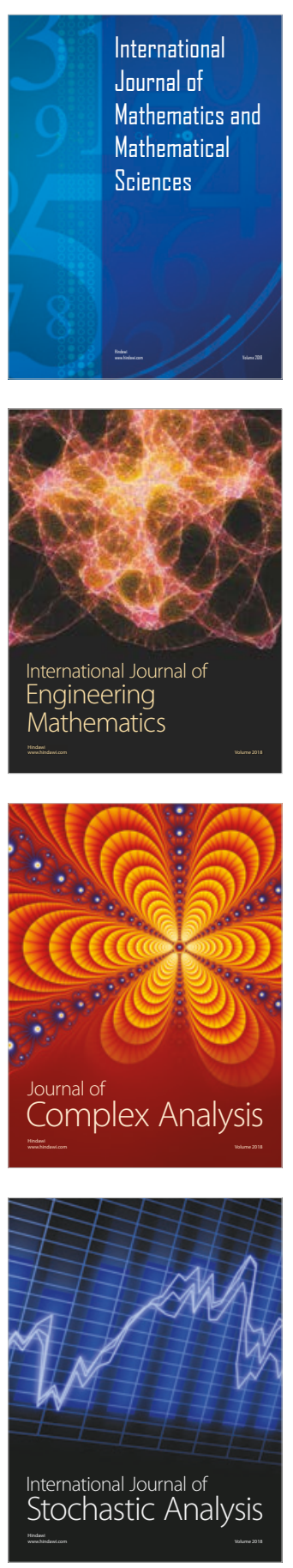
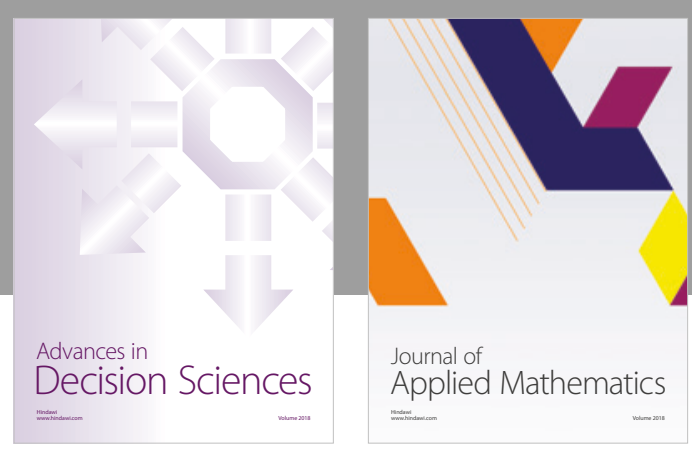

Journal of

Applied Mathematics
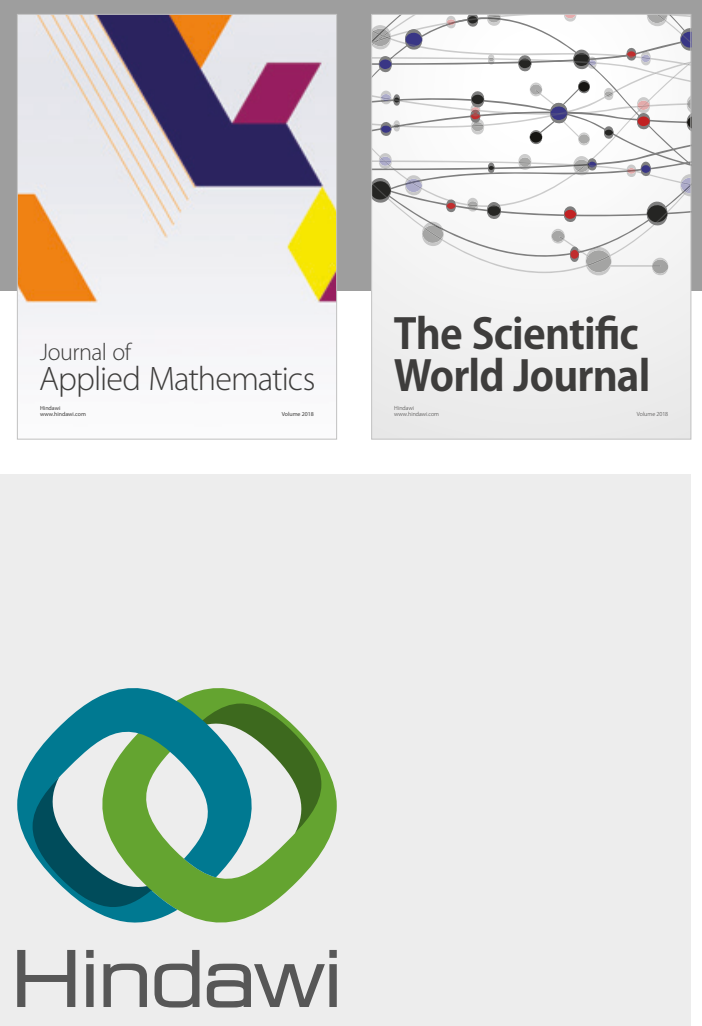

Submit your manuscripts at

www.hindawi.com

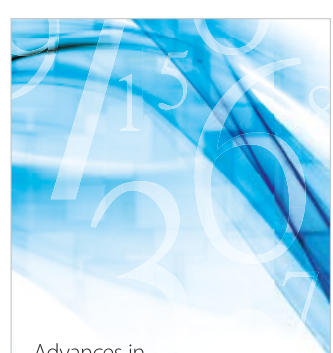

Advances in
Numerical Analysis
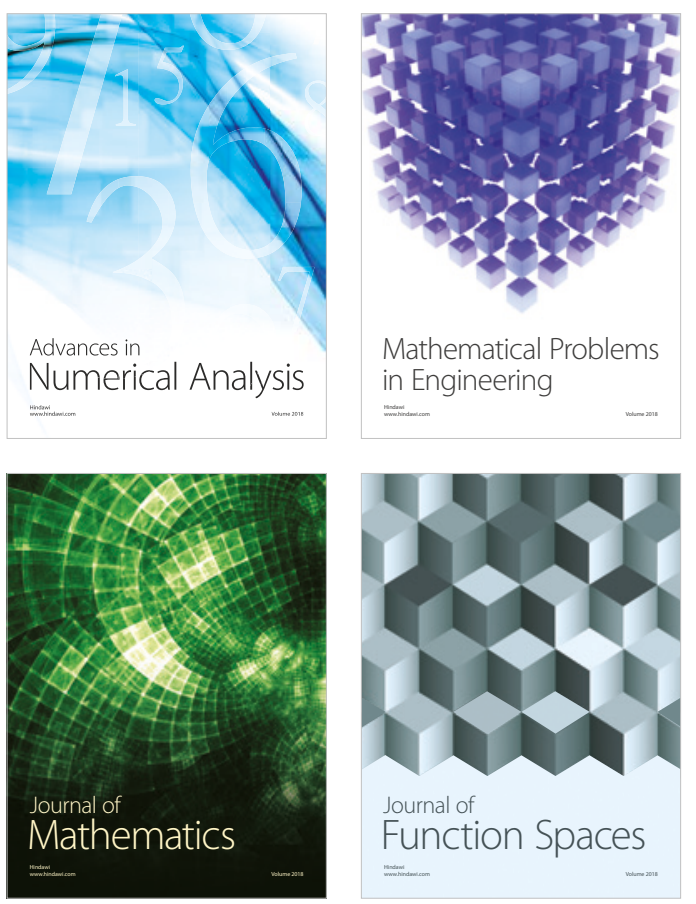

Mathematical Problems in Engineering

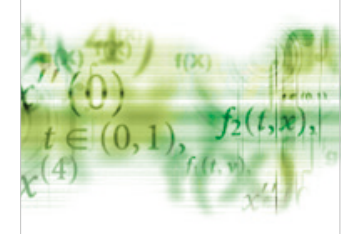

International Journal of

Differential Equations

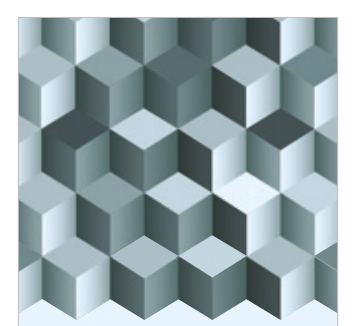

Journal of

Function Spaces

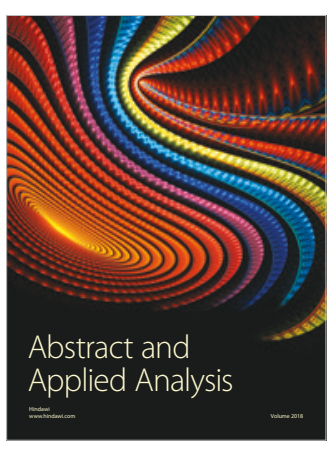

The Scientific

World Journal

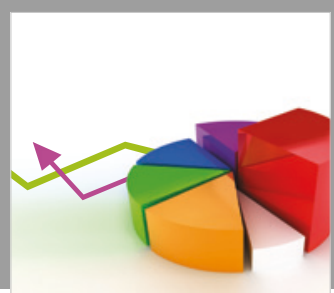

Journal of

Probability and Statistics
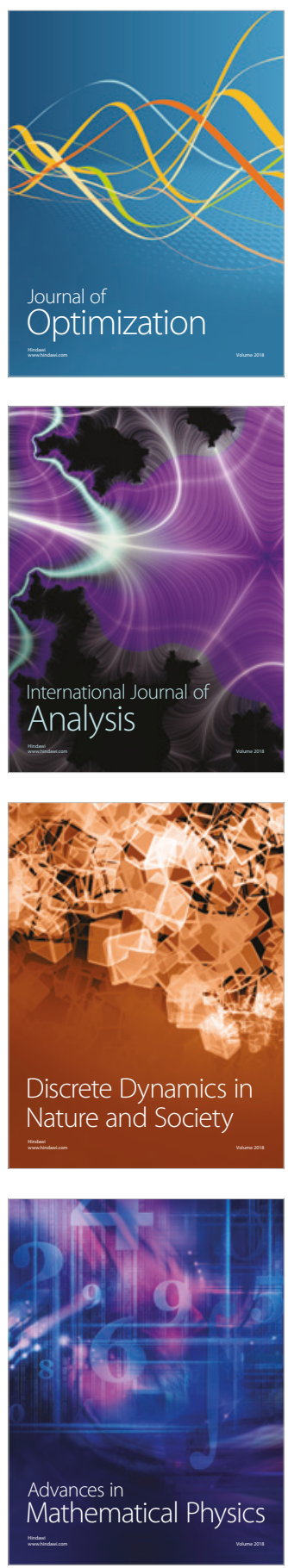